\title{
Association of OSAHS Hypoxia Indicators with Early Renal Injury and Serum Fibroblast Growth Factor 21 in Obese Type 2 Diabetic Patients
}

\author{
Weinan $\mathrm{Yu} \cdot \mathrm{Xiaoqing}$ Wang $\cdot$ Yaojun Ni $\cdot$ De Huai $\cdot$ Hairong Hao \\ Qingjun Li · Ziyu Liu · Surong Wen · Liang Cheng · Wen Hu
}

Received: April 11, 2019 / Published online: June 6, 2019

(C) The Author(s) 2019

\section{ABSTRACT}

Objective: This study aimed to explore the association of obstructive sleep apnea-hypopnea syndrome (OSAHS) hypoxia indicators with early renal injury and serum fibroblast growth factor 21 (FGF21) in obese type 2 diabetic patients.

Methods: A total of 109 obese patients with type 2 diabetes mellitus (T2DM) were recruited, including 70 males and 39 females, with an average age of $52.77 \pm 13.57$ years and average BMI of $29.08 \pm 4.36 \mathrm{~kg} / \mathrm{m}^{2}$. Overnight sleep monitoring was performed with a portable monitor to record respiratory

Enhanced Digital Features To view enhanced digital features for this article go to https://doi.org/10.6084/ m9.figshare.8166848.

Weinan Yu, Xiaoqing Wang and Yaojun Ni are co-first authors, and these authors contributed equally to this work.

W. Yu $\cdot$ X. Wang $\cdot$ H. Hao $\cdot$ Z. Liu $\cdot$ S. Wen .

L. Cheng · W. Hu ( $\square)$

Department of Endocrinology, Huai'an Hospital

Affiliated to Xuzhou Medical University and

Huai'an Second People's Hospital, Huai'an 223002, Jiangsu, China

e-mail: huwen787878@163.com

Y. Ni

Department of Cardiothoracic Surgery, Huai'an Hospital Affiliated to Nanjing Medical College and Huai'an First People's Hospital, Huai'an 223002, Jiangsu, China parameters [apnea-hypopnea index (AHI), oxygen desaturation index (ODI), lowest oxygen saturation $\left(\mathrm{LSaO}_{2}\right)$, mean oxygen saturation $\left(\mathrm{MSaO}_{2} / \mathrm{MPO}_{2}\right)$ and cumulative time of oxygen saturation $<90 \%(\mathrm{CT}<90 \%)$ ]. Ultrasonography was done to detect the quantitative liver fat content (LFC). The urine microalbumin and creatinine ratio (ACR) were determined by immunoturbidimetry. FGF21 was measured at baseline by enzyme-linked immunosorbent assay. Patients were divided into the proteinuria group $(n=42)$ and non-proteinuria group $(n=67)$. Correlation analysis and multivariate linear regression analysis were used to analyze the related data. In addition, patients were divided into the T2DM without OSAHS group $(n=16)$ and T2DM with OSAHS group $(n=93)$ according to the AHI value. The correlation analysis was used to assess the relationship between FGF21 and clinical variables.

\footnotetext{
D. Huai

Department of Otolaryngology, Huai'an Hospital Affiliated to Xuzhou Medical University and

Huai'an Second People's Hospital, Huai'an 223002, Jiangsu, China

Q. Li

Department of Endocrinology, Jiangning Hospital Affiliated to Nanjing Medical University, Nanjing 211100, Jiangsu, China
} 
Results: (1) ACR positively correlated with waist circumference (WC), AHI, ODI, CT $<90 \%$ and LFC, but negatively with $\mathrm{MSaO}_{2}$ and $\mathrm{LSaO}_{2}$. (2) AHI, ODI, CT $<90 \%$ and LFC were independent risk factors for ACR, $\mathrm{LSaO}_{2}$ and $\mathrm{MSaO}_{2}$ was a protective factor. (3) Serum FGF21 decreased in the OSAHS group compared with the non-OSAHS group. After adjustment for age, WC and TG, FGF21 correlated negatively with AHI, but positively with $\mathrm{MSaO}_{2}$.

Conclusions: AHI, ODI, CT $<90 \%$ and LFC are independent risk factors for ACR. FGF21 is associated with hypoxia indicators, and improving OSAHS status and reducing liver fat content may be helpful for the prevention and treatment of early diabetic nephropathy (DN).

Clinical Trial Registration Number: ChiCTRIOR-15006225.

Keywords: Fibroblast growth factor 21; Obesity; Obstructive sleep apnea-hypopnea syndrome; Type 2 diabetes mellitus; Urine microalbumin and creatinine ratio

\section{INTRODUCTION}

$\mathrm{DN}$, an important cause of end-stage renal diseases (ESRD) worldwide, has caused an enormous social and economic burden [1]. The progression of $\mathrm{DN}$ is variable, depending largely on the blood pressure (BP), obesity, metabolic control and other risk factors [2]. However, some patients may progress rapidly to ESRD despite active treatments [3], suggesting some unknown risk factors related to the development or progression of DN. Hence, it is critical to investigate some new risk factors of $\mathrm{DN}$.

OSAHS refers to repeated apnea and/or hypopnea in sleep due to recurrent collapse or obstruction of the upper airway of multiple causes. It is characterized by nocturnal hypoxemia, hypercapnia, arousal and sleeping structural disorder [4]. Currently, polysomnography is the gold standard in the diagnosis of OSAHS. The parameters used for the assessment of OSAHS include AHI, ODI, $\mathrm{LSaO}_{2}, \mathrm{MSaO}_{2}$ and $\mathrm{CT}<90 \%$. They can objectively and comprehensively evaluate the nighttime hypoxia of OSAHS subjects. AHI and nocturnal oxygen saturation are the main indicators in the diagnosis of OSAHS and the assessment of its severity [5]. Epidemiologic studies have shown that the prevalence of OSAHS in the hospitalized patients with T2DM is about 60\%, especially in those with obesity and poor glycemic control [6]. Furthermore, OSAHS has been shown to affect the glycemic control [7] and BP [8], contributing to the diabetic vascular complications. In addition to its effects on the glycemic control and hypertension, OSAHS and concomitant intermittent hypoxemia (IH) can trigger shared pathophysiologic pathways mediating the development of DM vascular complications including activation of oxidative and inflammatory pathways [9]. To date, a variety of studies have shown that the prevalence of OSAHS significantly increases in ESRD patients undergoing hemodialysis and that hypoxic-related indicators in hemodialysis patients are significantly higher than in nonESRD patients [10]. However, few studies have been conducted to investigate the relationship between OSAHS and DN in the early stage.

FGF21, a new member of the FGF family initially isolated from a mouse embryo, is involved in a variety of cellular functions including cell survival, differentiation, mitosis and angiogenesis [11]. Kim et al. [10] found that FGF21 was able to improve insulin resistance and prevent kidney damage by improving systemic metabolic changes and anti-fibrosis in DN. Moreover, administration of FGF21 may prevent diabetic-induced kidney injury by reducing lipid accumulation and inhibiting inflammation, oxidative stress and fibrosis [12]. Therefore, FGF21 is related to the suppression of inflammation and oxidative stress and may confer protection against the risk of DN. However, little is known about the relationship between OSAHS and FGF21 in T2DM patients.

The present study aimed to explore the association of OSAHS with early renal injury and serum FGF21 in obese T2DM patients in order to identify the possible serologic markers of early renal injury in patients with T2DM and OSAHS. Furthermore, timely intervention in high-risk patients with renal injury will delay or avoid the occurrence and development of DN. 


\section{METHODS}

\section{Patients}

A total of 109 patients with T2DM were recruited from November 2016 to November 2017 at the Second Huai'an Hospital, Affiliated Hospital of Xuzhou Medical College in Huai'an (Jiangsu, China).

Inclusion criteria were: (1) patients were diagnosed with T2DM based on the Guideline for the Prevention and Treatment of T2DM in China [13]; (2) according to the Guideline for Prevention and Treatment of Overweight and Obesity in Adults of China, overweight was defined as a body mass index (BMI) $>24 \mathrm{~kg} / \mathrm{m}^{2}$ [14]; (3) patients were 18-80 years old; (4) patients were able to perform self-care and instrumental activities of daily living without difficulties or need for help; (5) patients were able to provide self-reported data and informed consent.

Exclusion criteria were: T1DM, secondary DM, immune diseases, hepatitis, pregnancy, acute complications of DM (diabetic ketoacidosis and nonketotic hyperosmolar coma), central and mixed OSAHS, acute and chronic infection, neuropsychiatric disease, obvious upper airway obstruction, acute cardiovascular and cerebrovascular disease, severe liver and kidney dysfunction and hypothyroidism, gout, tumor or another serious disease. This study was approved by the Ethics Committee of the Second Huai'an People's Hospital, and informed consent was obtained from patients and their family members before the study.

\section{Sleep-Related Information}

All the patients received full polysomnography (PSG) over night with a Compumedics E 3142 polysomnography device (Compumedics Inc., Melbourne, Australia). AHI, ODI, $\mathrm{LSaO}_{2}, \mathrm{MSaO}_{2}$ and CT $<90 \%$ were detected. The patient did not use sedatives on the day of monitoring and did not drink tea, coffee or wine. OSAHS was diagnosed according to the Guideline for the Diagnosis and Treatment of OSAHS developed by the Chinese Medical Association Respiratory
Diseases Society: in 7-h sleep, the recurrence of apnea and hypopnea is $>30$ times or the AHI is $\geq 5$ times/h [5]. In addition, patients were divided into the T2DM without OSAHS group (non-OSAHS; $n=16$ ) and the T2DM with OSAHS group (OSAHS; $n=93$ ) according to the AHI.

\section{DN Assessment}

ACR is considered a sensitive indicator of early DN. In our study, patients were divided into the proteinuria group $(n=42)$ and non-proteinuria group ( $n=67)$ according to the 2014 criteria provided by the American Diabetes Association (IDA) and the American Kidney Foundation (NKF): urinary albumin/creatinine ratio $(\mathrm{ACR}) \geq 30 \mathrm{mg} / \mathrm{g}$ [15]. The estimated glomerular filtration rate (eGFR) was calculated based on the serum creatinine level by using the Chronic Kidney Disease Epidemiology Collaboration (CKD-EPI) equation [16].

\section{Detection of Liver Fat Content (LFC)}

Hepatic ultrasonography was performed in all patients by an experienced physician using an Aloka Prosound a6 scanner (Hitachi Aloka, Japan) with a $3-\mathrm{MHz}$ probe. The parameters used were as follows: the gain was $77 \mathrm{~dB}$ and depth was $15 \mathrm{~cm}$, which were standardized using a tissue-mimicking phantom before measurement. The ultrasound hepatic/renal echointensity ratio $(\mathrm{H} / \mathrm{R})$ and ultrasound hepatic echo-intensity attenuation rate (HA) were obtained from ordinary ultrasound images using the NIH image software (Image J 1.41o, National Institutes of Health, USA). LFC was measured according to a previous report [17].

\section{Covariates}

Baseline characteristics such as age, gender, duration of diabetes, history of smoking, history of drinking, hypertension and history of coronary heart disease were collected by reviewing medical records. Individuals were considered hypertensive if the average systolic blood pressure (SBP) was $\geq 140 \mathrm{mmHg}$, diastolic blood 
pressure (DBP) was $\geq 90 \mathrm{mmHg}$, or the use of pressure-lowering medications in the past year was reported. Waist circumference (WC) measurement: WC was measured in the standing position. The patient was asked to breathe naturally, and the distance between the midpoint of the anterior superior iliac spine and the lowest point of the lower rib bone around the abdomen was measured in $\mathrm{cm}$. BMI was computed as the ratio of weight to the square of height $\left(\mathrm{kg} / \mathrm{m}^{2}\right)$. Current smokers reported smoking at least one cigarette per day over the past month. Drinking was defined as drinking at least once weekly for nearly 1 month. BP was measured after resting for $10 \mathrm{~min}$. After overnight fasting (at least $10 \mathrm{~h}$ ), blood samples were collected from the antecubital vein between 8:00 and 10:00 a.m. Fasting glucose (FPG), total cholesterol (TC), triglycerides (TG), low-density lipoprotein cholesterol (LDL-C), high-density lipoprotein cholesterol (HDL-C), creatinine (CREA), urea nitrogen (BUN) and uric acid (UA) were measured with standardized procedures. HbA1c was measured by high-performance liquid chromatography (Variant II and D-10 Systems, Bio-Rad Laboratories Inc, Hercules, CA, USA). Enzyme-linked immunosorbent assay (human FGF21 ELISA kit, MBS177299, San Diego, CA, USA) was used to determine the plasma FGF21.

\section{Statistical Analysis}

All statistical analyses were performed using SPSS version 19.0 (SPSS Inc, Chicago, IL, USA). Each variable was tested for normal distribution using the Kolmogorov-Smirnov test. Abnormally distributed variables were analyzed with the Mann-Whitney $U$ test between the two groups. Quantitative data are presented as mean \pm standard deviation (SD) or percentage and median (25th and 75th percentiles). Student's $t$ test was used for comparing normally distributed variables and the Mann-Whitney $U$ test for abnormally distributed variables. Qualitative data are expressed as relative numbers, and comparison between groups was performed with the chi-square test. Spearman correlation analysis or partial correlation analysis was used for correlation analysis. A stepwise multiple regression analysis was done to investigate the independent variables associated with ACR. A value of $P<0.05$ was considered statistically significant.

\section{RESULTS}

\section{Baseline Information}

As shown in Table 1, a total of 109 patients were initially recruited, including 70 males and 39 females, with the average age of $52.77 \pm 13.57$ years and average BMI of $29.08 \pm 4.36 \mathrm{~kg} / \mathrm{m}^{2}$. According to the ACR, patients were divided into the proteinuria group $(n=42)$ and non-proteinuria group $(n=67)$. The average ACR in the proteinuria group was $4.8 \mathrm{mg} / \mathrm{mmol}$ and $1.2 \mathrm{mg} / \mathrm{mmol}$ in the nonproteinuria group. Several characteristics including male gender, hypertension, Scr, eGFR, LFC, AHI, ODI, $\mathrm{MPO}_{2}$ and $\mathrm{CT}<90 \%$, were significantly different between the two groups $(P<0.05)$. There were no significant differences in other indicators between the two groups $(P<0.05)$.

\section{Correlations of ACR with Clinical and Biochemical Parameters}

As shown in Table 2, the associations of age, FPG, TC, TG (logarithmic conversion), eGFR (logarithmic conversion), AHI, ODI, $\mathrm{LSaO}_{2}$ (logarithmic conversion), $\mathrm{MSaO}_{2}$ (logarithmic conversion) and CT $<90 \%$ (logarithmic conversion) with ACR were assessed. Results showed ACR positively correlated with WC, AHI, ODI, CT $<90 \%$ and LFC, but negatively with $\mathrm{MSaO}_{2}$ and $\mathrm{LSaO}_{2}(P<0.05)$.

\section{Multivariate Linear Regression Analysis of Hypoxia-Related Indicators and ACR}

ACR as a dependent variable and age, gender and duration of diabetes, SBP, DBP, WC, FPG, TC, TG, HDL-C, LDL-C, CREA, UA, LFC, AHI or ODI or $\mathrm{LSaO}_{2}$ or $\mathrm{MSaO}_{2}$ or CT $<90 \%$ as independent variables were employed to establish a 
Table 1 Characteristics of subjects in the proteinuria group and non-proteinuria group

\begin{tabular}{|c|c|c|c|c|}
\hline Variables & $\begin{array}{l}\text { Non-proteinuria group } \\
(n=67)\end{array}$ & $\begin{array}{l}\text { Proteinuria group } \\
(n=42)\end{array}$ & Statistics & $P$ \\
\hline Male, $n(\%)$ & $38(56.7)$ & $32(76.2)$ & $4.261 \mathrm{a}$ & 0.043 \\
\hline Age (years) & $51.1 \pm 14.1$ & $54.9 \pm 13.2$ & $-1.387 \mathrm{~b}$ & 0.168 \\
\hline History of hypertension, $n(\%)$ & $31(46.3)$ & $28(66.7)$ & $4.326 \mathrm{a}$ & 0.049 \\
\hline History of smoking, $n(\%)$ & $11(16.4)$ & $11(26.2)$ & $1.530 \mathrm{a}$ & 0.230 \\
\hline History of drinking, $n$ (\%) & $3(4.5)$ & $5(11.9)$ & $2.026 \mathrm{a}$ & 0.256 \\
\hline Duration of diabetes (years) & $3(1,10)$ & $8(2.8,13)$ & $1.342 \mathrm{c}$ & 0.055 \\
\hline BMI $\left(\mathrm{kg} / \mathrm{m}^{2}\right)$ & $28(25.3,31.4)$ & $29.1(26.9,33.0)$ & $1.184 \mathrm{c}$ & 0.121 \\
\hline $\mathrm{WC}(\mathrm{cm})$ & $93.8 \pm 9.3$ & $98.1 \pm 8.3$ & $-2.384 b$ & 0.019 \\
\hline $\mathrm{FPG}(\mathrm{mmol} / \mathrm{l})$ & $9.7(7.7,12.1)$ & $8.7(6.8,12.2)$ & $0.827 \mathrm{c}$ & 0.501 \\
\hline HbAlc (\%) & $8.1(6.7,10.7)$ & $8.7(7.1,10.1)$ & $0.563 \mathrm{c}$ & 0.909 \\
\hline $\mathrm{SBP}(\mathrm{mmHg})$ & $131.8 \pm 12.2$ & $134.8 \pm 12.2$ & $-1.216 \mathrm{~b}$ & 0.227 \\
\hline $\mathrm{DBP}(\mathrm{mmHg})$ & $77.9 \pm 7.8$ & $80.1 \pm 9.2$ & $-1.353 b$ & 0.179 \\
\hline $\mathrm{TC}(\mathrm{mmol} / \mathrm{l})$ & $4.9 \pm 1.2$ & $4.8 \pm 1.2$ & $0.780 \mathrm{~b}$ & 0.437 \\
\hline $\mathrm{TG}(\mathrm{mmol} / \mathrm{l})$ & $1.6(1.1,2.9)$ & $2.0(1.6,3.6)$ & $1.383 \mathrm{c}$ & 0.044 \\
\hline LDL-C (mmol/l) & $2.7 \pm 0.8$ & $2.5 \pm 0.8$ & $1.396 \mathrm{~b}$ & 0.166 \\
\hline HDL-C $(\mathrm{mmol} / \mathrm{l})$ & $1.2 \pm 0.3$ & $1.2 \pm 0.3$ & $-0.067 b$ & 0.946 \\
\hline $\operatorname{Scr}(\mu \mathrm{mol} / \mathrm{l})$ & $63.9(50.0,70.3)$ & $65.81(58.3,84.1)$ & $1.322 \mathrm{c}$ & 0.061 \\
\hline BUN (mmol/l) & $5.4(4.3,6.3)$ & $5.6(4.7,7.6)$ & $1.087 \mathrm{c}$ & 0.188 \\
\hline $\mathrm{UA}(\mu \mathrm{mol} / \mathrm{l})$ & $284.7(229.0,354.2)$ & $294.6(239.7,391.2)$ & $0.740 \mathrm{c}$ & 0.644 \\
\hline eGFR (ml/min) & $108.2(95.8,120.2)$ & $100.5(90.7,109.9)$ & $1.430 \mathrm{c}$ & 0.033 \\
\hline LFC (\%) & $15.35 \pm 5.96$ & $19.2 \pm 6.8$ & $-3.314 b$ & 0.002 \\
\hline AHI (times/h) & $22.15 \pm 16.56$ & $31.2 \pm 20.0$ & $-2.453 b$ & 0.016 \\
\hline ODI (times/h) & $19.18 \pm 15.00$ & $28.6 \pm 17.5$ & $-2.892 b$ & 0.005 \\
\hline $\mathrm{LSaO}_{2}(\%)$ & $80(73,85)$ & $76.0(64.8,81.3)$ & $1.370 \mathrm{c}$ & 0.047 \\
\hline $\mathrm{MPO}_{2}(\%)$ & $96(95,97)$ & $95(93.8,96.0)$ & $1.567 \mathrm{c}$ & 0.015 \\
\hline CT $<90 \%(\%)$ & $0.94(0.3,2.0)$ & $3.4(0.6,7.1)$ & $1.782 \mathrm{c}$ & 0.003 \\
\hline FGF21 (pg/ml) & $175.8(139.6,226.6)$ & $0.9(147.6,238.1)$ & $0.459 \mathrm{c}$ & 0.984 \\
\hline OSAHS, $n(\%)$ & $55(82.1)$ & $38(90.1)$ & $1.450 \mathrm{a}$ & 0.229 \\
\hline
\end{tabular}


Table 1 continued

\begin{tabular}{lllll}
\hline Variables & $\begin{array}{l}\text { Non-proteinuria group } \\
(\boldsymbol{n}=\mathbf{6 7})\end{array}$ & $\begin{array}{l}\text { Proteinuria group } \\
(\boldsymbol{n}=\mathbf{4 2})\end{array}$ & Statistics & $\boldsymbol{P}$ \\
\hline $\mathrm{ACR}(\mathrm{mg} / \mathrm{mmol})$ & $1.2(0.8,1.7)$ & $4.8(3.5,14.03)$ & $5.0814 \mathrm{c}$ & 0.001 \\
\hline
\end{tabular}

All variables are expressed as $n$ (\%) for categorical data or mean \pm SD or medians (25th and 75 th percentiles) for continuous data with or without normal distribution, respectively

Serum creatinine, $1 \mathrm{mg} / \mathrm{dl}=88.41 \mu \mathrm{mol} / 1$

$B M I$ body mass index, $W C$ waist circumference, $F P G$ fasting glucose, $H b A 1 c$ hemoglobin A1C, $S B P$ systolic blood pressure, $D B P$ diastolic blood pressure, $T C$ total cholesterol, $T G$ triglycerides, $H D L-C$ high-density lipoprotein cholesterol, $L D L-C$ low-density lipoprotein cholesterol, $H D L-C$ high-density lipoprotein cholesterol, $S c r$ serum creatinine, $B U N$ urea nitrogen, $U A$ uric acid, $e G F R$ estimated glomerular filtration rate, $L F C$ liver fat content, $A H I$ apnea-hypopnea index, $O D I$ oxygen desaturation index, $\mathrm{LSaO}_{2}$ minimum oxygen saturation, $\mathrm{MPO}_{2}$ average oxygen saturation, $C T 90 \%$ cumulative time of $\mathrm{SPO}_{2}$ $<90 \%, F G F 21$ fibroblast growth factor 21, OSAHS obstructive sleep apnea-hypopnea syndrome

Table 2 Correlation between ACR and various indicators

\begin{tabular}{|c|c|c|}
\hline Variable & $r_{\mathrm{s}}$ & $P$ value \\
\hline Age (years) & 0.173 & 0.072 \\
\hline $\mathrm{WC}(\mathrm{cm})$ & 0.189 & 0.049 \\
\hline FPG $(\mathrm{mmol} / \mathrm{l})$ & -0.089 & 0.360 \\
\hline $\mathrm{TC}(\mathrm{mmol} / \mathrm{l})$ & -0.126 & 0.193 \\
\hline $\operatorname{lgTG}(\mathrm{mmol} / \mathrm{l})$ & 0.076 & 0.430 \\
\hline $\operatorname{lgeGFR}(\mathrm{ml} / \mathrm{min})$ & -0.235 & 0.014 \\
\hline AHI (times/h) & 0.253 & 0.008 \\
\hline ODI (times/h) & 0.291 & 0.004 \\
\hline $\operatorname{lgLSaO}_{2}(\%)$ & -0.227 & 0.018 \\
\hline $\operatorname{lgMPO}_{2}(\%)$ & -0.304 & 0.001 \\
\hline $\operatorname{lgCT}<90 \%(\%)$ & 0.217 & 0.012 \\
\hline LFC (\%) & 0.291 & 0.002 \\
\hline FGF21 $(\mathrm{pg} / \mathrm{ml})$ & 0.082 & 0.394 \\
\hline
\end{tabular}

$S B P$ systolic blood pressure, $D B P$ diastolic blood pressure, $W C$ waist circumference, $F P G$ fasting glucose, $H b A l c$ hemoglobin A1C, $T C$ total cholesterol, $T G$ triglyceride, $H D L-C$ high-density lipoprotein cholesterol, $L D L-C$ lowdensity lipoprotein cholesterol, $H D L-C$ high-density lipoprotein cholesterol, $e G F R$ estimated glomerular filtration rate, $U A$ uric acid, $A H I$ apnea-hypopnea index, $O D I$ oxygen desaturation index, $\mathrm{LSaO}_{2}$ minimum oxygen saturation, $\mathrm{MPO}_{2}$ average oxygen saturation, CT90\% cumulative time of $\mathrm{SPO}_{2}$ below 90\%, $L F C$ liver fat content, FGF21 fibroblast growth factor 21 multivariate linear stepwise regression model. Results showed AHI, ODI, CT $<90 \%$ and LFC were independent risk factors for ACR, but $\mathrm{LSaO}_{2}$ and $\mathrm{MSaO}_{2}$ were protective factors $(P<0.05$, Fig. 1).

\section{Correlation between Serum FGF21 and Hypoxia-Related Indicators}

Patients were divided into the non-OSAHS group $(n=16)$ and OSAHS group $(n=93)$ according to the AHI. At baseline, serum FGF21 in the OSAHS group was significantly lower than in the non-OSAHS group $(P<0.05$, Table 3). Correlation analysis showed that serum FGF21 was negatively related to AHI and ODI in the OSAHS group, while there was no close correlation of serum FGF21 with the OSAHS index in the non-OSAHS group $(P<0.05$, Table 4$)$. After adjusting for age, WC and TG, FGF21 was negatively related to AHI, but positively with $\mathrm{MSaO}_{2}(P<0.05$, Table 4$)$.

\section{DISCUSSION}

$\mathrm{DN}$ is a main cause of ESRD and closely related to various factors such as obesity, genetics, hemodynamics, hyperglycemia and oxidative stress, seriously affecting the health of patients and increasing mortality [2]. In recent years, OSAHS has been increasingly concerned because of its high prevalence in T2DN patients [18]. OSHAS is a chronic sleep disorder 


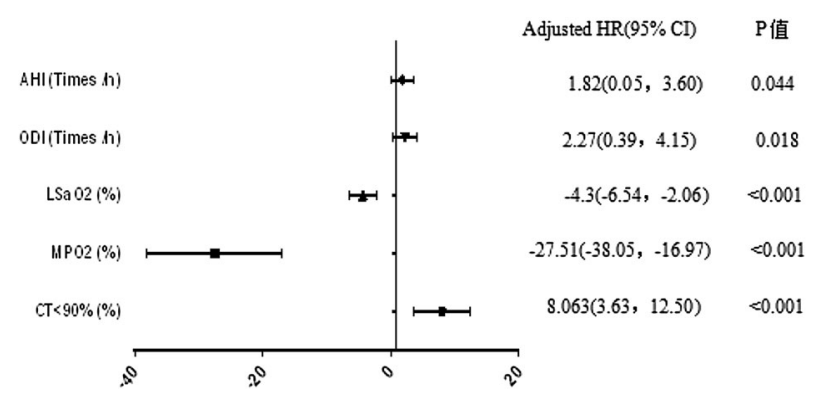

Fig. 1 Multivariate logistic regression analysis on influencing factors of ACR. $C I$ confidence interval, $A H I$ apneahypopnea index, $\mathrm{ODI}$ oxygen desaturation index, $\mathrm{LSaO}_{2}$ the minimum oxygen saturation, $\mathrm{MPO}_{2}$ average oxygen saturation, CT90\% cumulative time of $\mathrm{SPO}_{2}<90 \%$. Adjusted for age, gender and duration of diabetes characterized by episodic complete or partial upper airway obstruction causing $\mathrm{IH}$ and sleep fragmentation. Studies have shown that, besides the influence on glycemic control and hypertension, OSAHS and concomitant IH may also trigger shared pathophysiologic pathways (including oxidative and inflammatory pathways) mediating DN [9]. Serum FGF21 is a new metabolic regulator that can improve insulin resistance and maintain glucose homeostasis. In addition, it is also involved in the inhibition of inflammation and oxidative stress and has an anti-fibrotic effect on various diseases [19]. Therefore, to explore the association of OSAHS with early renal injury and serum FGF21 may help prevent and delay the development of DN.

ACR serves as a sensitive indicator of early kidney injury in DN [20]. Studies have shown that ACR is an independent predictor of cardiovascular events in subjects with or without cardiovascular risk factors, and treatments that reduce albumin excretion can also slow renal failure and improve cardiovascular risk [21]. In our study, ACR was used as a diagnostic criterion for early $\mathrm{DN}$.

Currently, little is known about the relationship between OSAHS and early DN, and the available findings are conflicting. Our results showed there were significant differences in the AHI, ODI, $\mathrm{LSaO}_{2}, \mathrm{MSaO}_{2}, \mathrm{CT}<90 \%$, eGFR and LFC between DN patients and non-DN patients. Correlation analysis showed ACR positively correlated with WC, AHI, ODI, CT $<90 \%$ and LFC, but negatively with $\mathrm{MSaO}_{2}$ and $\mathrm{LSaO}_{2}$. This was consistent with the results from a recent study: a significant association was found between AHI category and aACR with the AHI $\geq 30$ having the highest aACR [22]. Buyukaydin et al. [23] examined the clinical parameters of 52 patients with T2DM and OSAHS; their results showed there was no significant difference in the AHI between the micro-albuminuria $(9.0 \pm 14.6$ events/h) group and non-micro-albuminuria (10.5 \pm 10.0 events/h) group. This might be ascribed to the small sample size $(n=52)$ and the relatively high proportions of females (72\%) because OSAHS is more common in men. It is necessary to carry out more large sample and multicenter studies to confirm our findings.

ACR is known as a sensitive indicator of DN. Our results showed ACR positively correlated with a partial hypoxia index, eGFR and LFC, but negatively with $\mathrm{MSaO}_{2}$ and $\mathrm{LSaO}_{2}$. However, there was no correlation of ACR with FPG, HbA1c, TG and LDL-C, which was different from the results reported by Taleb et al. [24]. On one hand, this may be due to the relatively small sample size of our study, and thus the correlation between variables could not be identified; on the other hand, our results might be biased by sample selection, because the blood glucose in our study was well controlled and the blood lipid was also within the normal range. It is well known that the kidney is rich in blood and has high perfusion, which makes the kidney sensitive to hypoxic injury, while OSAHS is characterized by chronic IH. In early renal injury, hypoxia accounts for more than the changes in blood glucose and blood lipid. In further analysis, ACR served as a dependent variable, and OSAHS-related parameters and 
Table 3 Characteristics in the T2DM with OSAHS group and T2DM without OSAHS group

\begin{tabular}{|c|c|c|c|c|}
\hline Variable & $\begin{array}{l}\text { Non-OSAHS group } \\
(n=16)\end{array}$ & $\begin{array}{l}\text { OSAHS group } \\
(n=93)\end{array}$ & Statistics & $\boldsymbol{P}$ \\
\hline Age (years) & $47.8 \pm 11.6$ & $53.4 \pm 14.0$ & $-1.506 b$ & 0.135 \\
\hline Male, $n(\%)$ & $8(50.0)$ & $62(66.7)$ & $1.650 \mathrm{a}$ & 0.260 \\
\hline History of hypertension, $n(\%)$ & $8(50.0)$ & $51(66.7)$ & $0.129 \mathrm{a}$ & 0.720 \\
\hline History of smoking, $n(\%)$ & $1(6.3)$ & $21(22.6)$ & $1.360 \mathrm{a}$ & 0.244 \\
\hline History of drinking, $n(\%)$ & $1(6.3)$ & $7(7.5)$ & $0.034 \mathrm{a}$ & 0.854 \\
\hline Duration of diabetes (years) & $2.0(0.1,5.0)$ & $5(1,10.5)$ & $0.976 c$ & 0.297 \\
\hline $\operatorname{BMI}\left(\mathrm{kg} / \mathrm{m}^{2}\right)$ & $25.4(24.5,27.1)$ & $28.9(26.8,32.3)$ & $1.890 \mathrm{c}$ & 0.002 \\
\hline $\mathrm{WC}(\mathrm{cm})$ & $87.3 \pm 5.8$ & $96.8 \pm 8.9$ & $-4.098 b$ & $<0.001$ \\
\hline FPG $(\mathrm{mmol} / \mathrm{l})$ & $8.9(7.2,11.2)$ & $9.6(7.1,12.2)$ & $0.618 c$ & 0.839 \\
\hline HbAlc $(\%)$ & $8.0(6.7,10.0)$ & $8.4(6.9,10.7)$ & $0.554 \mathrm{c}$ & 0.919 \\
\hline SBP $(\mathrm{mmHg})$ & $132.9 \pm 14.3$ & $133.0 \pm 12.0$ & $-0.031 b$ & 0.975 \\
\hline $\mathrm{DBP}(\mathrm{mmHg})$ & $80.6 \pm 8.7$ & $78.4 \pm 8.4$ & $0.979 b$ & 0.330 \\
\hline $\mathrm{TC}(\mathrm{mmol} / \mathrm{l})$ & $4.7 \pm 0.9$ & $4.9 \pm 1.3$ & $-0.444 b$ & 0.658 \\
\hline $\mathrm{TG}(\mathrm{mmol} / \mathrm{l})$ & $1.9(1.0,3.4)$ & $1.8(1.3,3.0)$ & $1.383 c$ & 0.044 \\
\hline LDL-C (mmol/l) & $2.6 \pm 0.9$ & $2.7 \pm 0.8$ & $-0.256 b$ & 0.799 \\
\hline HDL-C (mmol/l) & $1.1 \pm 0.3$ & $1.2 \pm 0.3$ & $-0.910 b$ & 0.365 \\
\hline $\operatorname{Scr}(\mu \mathrm{mol} / \mathrm{l})$ & $60.0(44.0,68.6)$ & $64.5(52.8,74.3)$ & $0.725 c$ & 0.669 \\
\hline BUN (mmol/l) & $4.46(4.27,6.33)$ & $5.55(4.68,6.69)$ & $1.284 \mathrm{c}$ & 0.074 \\
\hline $\mathrm{UA}(\mu \mathrm{mol} / \mathrm{l})$ & $278.7(231.7,376.4)$ & $294.6(234.6,356.6)$ & $0.544 c$ & 0.929 \\
\hline eGFR (ml/min) & $105.4(98.5,120.7)$ & $103.2(92.4,116.3)$ & $1.001 \mathrm{c}$ & 0.269 \\
\hline $\mathrm{ACR}(\mathrm{mg} / \mathrm{mmol})$ & $1.27(0.97,3.5)$ & $1.96(1.00,4.29)$ & $0.904 \mathrm{c}$ & 0.387 \\
\hline LFC (\%) & $11.1 \pm 4.4$ & $17.8 \pm 6.4$ & $-4.065 b$ & $<0.001$ \\
\hline FGF21 (pg/ml) & $218.7(195.9,307.5)$ & $167.6(141.0,221.9)$ & $1.770 \mathrm{c}$ & 0.004 \\
\hline
\end{tabular}

All variables are expressed as $n$ (\%) for categorical data or as mean \pm SD or medians (25th and 75th percentiles) for continuous data with or without a normal distribution, respectively. $P$ values tested the overall difference among OSAHS classifications

Serum creatinine, $1 \mathrm{mg} / \mathrm{dl}=88.41 \mu \mathrm{mol} / 1$

$B M I$ body mass index, $W C$ waist circumference, $F P G$ fasting glucose, $H b A 1 c$ hemoglobin A1C, $S B P$ systolic blood pressure, $D B P$ diastolic blood pressure, $T C$ total cholesterol, $T G$ triglyceride, $H D L-C$ high-density lipoprotein cholesterol, $L D L-C$ low-density lipoprotein cholesterol, $H D L-C$ high-density lipoprotein cholesterol, $S c r$ serum creatinine, $B U N$ urea nitrogen, $U A$ uric acid, $e G F R$ estimated glomerular filtration rate, $A C R$ albumin/creatinine ratio., $L F C$ liver fat content, $F G F 21$ fibroblast growth factor 21

other clinical data were used as independent variables. Stepwise linear regression showed AHI and ODI were independent risk factors for ACR.
This was consistent with the results on the relationship between AHI and urinary microalbumin reported by Zhang et al. [25] and on the 
Table 4 Correlation between serum FGF21 and clinical indicators in the T2DM with OSAHS group and non-OSAHS group

\begin{tabular}{|c|c|c|c|c|c|c|}
\hline \multirow[t]{3}{*}{ Variable } & \multicolumn{4}{|c|}{ OSAHS group } & \multirow{2}{*}{\multicolumn{2}{|c|}{$\begin{array}{l}\text { Non-OSAHS group } \\
\text { FGF21 }\end{array}$}} \\
\hline & \multicolumn{2}{|l|}{$\overline{\text { FGF21 }}$} & \multicolumn{2}{|c|}{ FGF21 (adjustment for age, WC and TG) } & & \\
\hline & $\overline{r_{\mathrm{s}}}$ & $P$ & $\overline{r_{\mathrm{s}}}$ & $P$ & $\overline{r_{s}}$ & $P$ \\
\hline Age (years) & 0.229 & 0.028 & & & -0.331 & 0.210 \\
\hline $\mathrm{SBP}(\mathrm{mmHg})$ & -0.165 & 0.115 & -0.121 & 0.255 & 0.191 & 0.478 \\
\hline $\mathrm{DBP}(\mathrm{mmHg})$ & 0.080 & 0.443 & 0.105 & 0.324 & 0.065 & 0.812 \\
\hline WC $(\mathrm{cm})$ & -0.225 & 0.030 & & & -0.077 & 0.778 \\
\hline $\mathrm{FPG}(\mathrm{mmol} / \mathrm{l})$ & -0.005 & 0.959 & 0.005 & 0.959 & 0.531 & 0.034 \\
\hline HbAlc (\%) & 0.028 & 0.787 & 0.042 & 0.693 & 0.451 & 0.079 \\
\hline $\mathrm{TC}(\mathrm{mmol} / \mathrm{l})$ & -0.071 & 0.449 & -0.143 & 0.179 & -0.218 & 0.418 \\
\hline $\mathrm{TG}(\mathrm{mmol} / \mathrm{l})$ & -0.207 & 0.047 & & & 0.006 & 0.983 \\
\hline LDL-C (mmol/l) & -0.004 & 0.969 & -0.187 & 0.077 & 0.049 & 0.858 \\
\hline HDL-C $(\mathrm{mmol} / \mathrm{l})$ & -0.155 & 0.137 & -0.054 & 0.616 & -0.358 & 0.174 \\
\hline eGFR $\left(\mathrm{ml} / \mathrm{min} / 1.73 \mathrm{~m}^{2}\right)$ & -0.278 & 0.007 & -0.064 & 0.551 & 0.397 & 0.128 \\
\hline $\mathrm{UA}(\mu \mathrm{mol} / \mathrm{l})$ & -0.083 & 0.426 & 0.014 & 0.894 & -0.041 & 0.880 \\
\hline AHI (times/h) & -0.214 & 0.040 & -0.227 & 0.031 & -0.170 & 0.528 \\
\hline ODI (times/h) & -0.22 & 0.034 & -0.191 & 0.072 & 0.102 & 0.708 \\
\hline $\mathrm{LSaO}_{2}(\%)$ & 0.106 & 0.310 & 0.152 & 0.152 & -0.016 & 0.952 \\
\hline $\mathrm{MPO}_{2}(\%)$ & 0.176 & 0.091 & 0.209 & 0.049 & -0.399 & 0.126 \\
\hline $\mathrm{CT}<90 \%(\%)$ & -0.179 & 0.086 & -0.189 & 0.074 & -0.133 & 0.622 \\
\hline LFC (\%) & -0.052 & 0.621 & 0.044 & 0.679 & 0.032 & 0.905 \\
\hline $\mathrm{ACR}(\mathrm{mg} / \mathrm{mmol})$ & 0.092 & 0.380 & -0.102 & 0.339 & 0.235 & 0.380 \\
\hline
\end{tabular}

$S B P$ systolic blood pressure, $D B P$ diastolic blood pressure, $W C$ waist circumference, $F P G$ fasting glucose, $H b A 1 c$ hemoglobin A1C, $T C$ total cholesterol, $T G$ triglyceride, $H D L-C$ high-density lipoprotein cholesterol, $L D L-C$ low-density lipoprotein cholesterol, $H D L-C$ high-density lipoprotein cholesterol, $e G F R$ estimated glomerular filtration rate, $U A$ uric acid, $A H I$ apnea-hypopnea index, $O D I$ oxygen desaturation index, $\mathrm{LSaO}_{2}$ minimum oxygen saturation, $\mathrm{MPO}_{2}$ average oxygen saturation, $C T 90 \%$ the cumulative time of $\mathrm{SPO}_{2}<90 \%, L F C$ liver fat content, $A C R$ albumin/creatinine ratio

relationship between ODI and urinary microalbumin reported by Furukawa et al. [26]. However, few studies have been conducted to investigate the correlation of urinary albumin with $\mathrm{MSaO}_{2}$ and $\mathrm{LSaO}_{2}$. Our results showed $\mathrm{MSaO}_{2}$ was an independent risk factor for ACR, and $\mathrm{LSaO}_{2}$ was a protective factor. Therefore, we speculate that hypoxia is an independent risk factor for early renal injury in DM patients.
The exact mechanism between OSAHS and microalbuminuria is unclear. The possible mechanisms may be as follows: (1) The sleeprelated pathophysiologic changes in case of OSAHS may influence the glomerular endothelial function and the urinary albumin excretion. IH caused by OSAHS has been documented to cause oxidative stress and activation of the inflammatory pathways, leading to endothelial dysfunction [9]. In addition, chronic $\mathrm{IH}$ is 
associated with a three-fold increase in macroalbuminuria and a two-fold increase in microalbuminuria [26]. (2) The intermittent intra-renal hemodynamic changes due to recurrent sympathetic overdrive secondary to sleep fragmentation can cause ischemia and intra-renal reperfusion injury, leading to intrinsic renal injury [27]. A case report on OSAHS showed secondary focal glomerulosclerosis, and proteinuria improved completely after treatment with bi-level positive airway pressure in one patient [28]. (3) Hypoxia may directly affect the $\beta$-cell function and activate inflammatory molecular pathways to alter the insulin sensitivity. Overall, these mechanisms can elevate the insulin resistance in T2DM patients and affect other related factors of microalbuminuria.

In addition, the plasma FGF21 was detected in our study. Some studies have shown that FGF21 is elevated in DN patients and may be a predictor of DN [29]. A study [12] showed that serum FGF-21 increased in T2DM patients compared with non-diabetic controls, and it was significantly higher in patients with an elevated urinary albumin excretion rate. However, our results showed no significant difference in FGF21 between the proteinuria group and non-proteinuria group. It should be noted that most of the subjects in our study had OSAHS, and there were significant differences in OSAHS-related indicators between the two groups. Perhaps hypoxia-related indicators may affect the plasma FGF21 level. We further divided patients into the T2DM without OSAHS group and T2DM with OSAHS group. Results indicated that serum FGF21 in the T2DM with OSAHS group was significantly lower than in the T2DM without OSAHS group, and serum FGF21 in the T2DM with OSAHS group negatively correlated with AHI and ODI. However, serum FGF21 was not associated with OSAHS parameters in the non-OSAHS group. The specific mechanism is unclear. A study [30] about the effects of hypoxia on the FGF21 expression in Caco-2 cells indicates hypoxia significantly reduces FGF21 gene and protein expression, which is ascribed to the hypoxiainduced oxidative stress and mRNA stabilization. Therefore, we speculate that hypoxia may reduce the expression of serum FGF21 through some mechanisms, thus further affecting the occurrence and development of DN.

The novelties of this study were as follows: First, we systematically investigated the relationship between OSAHS indicators and ACR in obese patients with T2DM. Second, our results showed ACR had a significant correlation with LFC. Furthermore, although the eGFR between the proteinuria group and non-proteinuria group was within the normal range, eGFR in the proteinuria group decreased compared with the non-proteinuria group. This suggests that clinicians should not only pay attention to whether eGFR is in the normal range, but also dynamically monitor the changes in eGFR. Finally, our study showed serum FGF21 decreased in the OSAHS group compared with the non-OSAHS group, and serum FGF21 negatively correlated with AHI, but positively with $\mathrm{MSaO}_{2}$. Studies have shown that hypoxemia parameters may reflect the association of OSAHS with diabetic microvascular complications more sensitively than AHI, but the specific mechanism remains to be further explored.

There were several limitations in this study. First, this was a retrospective study and the sample size was relatively small. Second, only ACR was used as a criterion in our study, and the changes in GFR and glomerular pathology were not evaluated. Third, the causal relationship between OSAHS and DN was unclear. However, it is necessary to pay attention to the screening and diagnosis of OSAHS, especially in those with obesity and non-alcoholic fatty liver, which may benefit the treatment of microalbuminuria.

In conclusion, our study indicates that $\mathrm{AHI}$ and ODI are independent risk factors for ACR. Compared with non-OSAHS patients, serum FGF21 significantly reduces in T2DM with OSAHS patients, and it correlates with AHI and ODI. The specific interaction mechanism among hypoxia, FGF21 and DN remains unclear. In the future, more prospective studies with large sample size are needed to explore the effects of OSAHS on the urinary protein and eGFR in patients with T2DM and to investigate the relationships among serum FGF21, OSAHS and $\mathrm{DN}$ to provide more evidence for the prevention and treatment of $\mathrm{DN}$. 


\section{ACKNOWLEDGEMENTS}

The authors thank all participants for their cooperation and sample contribution.

Funding. This study was supported by the National Natural Science Foundation of China (81700776) and Key R\&D Program of Huaian City, Jiangsu Province (HAS201611-04). The study sponsor is also funding the journal's article processing charges.

Authorship. All named authors meet the International Committee of Medical Journal Editors (ICMJE) criteria for authorship for this article, take responsibility for the integrity of the work as a whole, and have given their approval for this version to be published.

Author Contributions. Weinan Yu, Xiaoqing Wang and Yaojun $\mathrm{Ni}$ are responsible for the conception and design of the article, analyzing and interpreting the results, writing papers and paper revisions; Wen $\mathrm{Hu}$, Weinan $\mathrm{Yu}$ and Yaojun Ni conducted feasibility analysis, participating in the writing of papers and paper revisions; Xiaoqing Wang, De Huai, Hairong Hao, Qingjun Li, Ziyu Liu and SuRong Wen participated in the implementation of the study and data collection; Xiaoqing Wang, De Huai and Liang Cheng conducted data collation and statistical processing; Wen $\mathrm{Hu}$ is responsible for the quality control, review and supervision of the article and is responsible for the overall article.

Disclosures. Weinan $\mathrm{Yu}$, Xiaoqing Wang, Yaojun Ni, De Huai, Hairong Hao, Qingjun Li, Ziyu Liu, Surong Wen, Liang Cheng and Wen $\mathrm{Hu}$ have nothing to declare.

Compliance with Ethics Guidelines. This study was approved by the Ethics Committee of the Second Huai'an People's Hospital, and informed consent was obtained from patients and their family members before the study. All procedures performed in studies involving human participants were in accordance with the ethical standards of the institutional research committee and with the 1964 Helsinki
Declaration and its later amendments or comparable ethical standards.

Data Availability. The datasets of our study are available from the corresponding author on reasonable request.

Open Access. This article is distributed under the terms of the Creative Commons Attribution-NonCommercial 4.0 International License (http://creativecommons.org/licenses/ by-nc/4.0/), which permits any noncommercial use, distribution, and reproduction in any medium, provided you give appropriate credit to the original author(s) and the source, provide a link to the Creative Commons license, and indicate if changes were made.

\section{REFERENCES}

1. Bakris GL. Recognition, pathogenesis, and treatment of different stages of nephropathy in patients with type 2 diabetes mellitus. Mayo Clin Proc. 2011;86:444-56.

2. Afghahi H, Cederholm J, Eliasson B, et al. Risk factors for the development of albuminuria and renal impairment in type 2 diabetesdthe Swedish National Diabetes Register (NDR). Nephrol Dial Transplant. 2011;26:1236-43.

3. Caliskan Y, Kiryluk K. Novel biomarkers in glomerular disease. Adv Chronic Kidney Dis. 2014;21:205-16.

4. Crawford JA, Montevecchi F, Vicini C, Magnuson JS. Transoral robotic sleep surgery: the obstructive sleep apnea-hypopnea syndrome. Otolaryngol Clin North Am. 2014;47(3):397-406.

5. He Quanzhen, Chen Baoyuan. Interpretation of the guidelines for the diagnosis and treatment of obstructive sleep apnea hypopnea syndrome (2011 revised edition) [J]. Chin J Tuberc Respir Dis. 2012;35(1):7-8.

6. Zhang P, Zhang R, Zhao F, et al. The prevalence and characteristics of obstructive sleep apnea in hospitalized patients with type 2 diabetes in China[J]. J Sleep Res. 2016;25(1):39-46.

7. Resnick HE, Redline S, Shahar E, et al. Diabetes and sleep disturbances: findings from the Sleep Heart Health Study. Diabetes Care. 2003;26:702-9. 
8. Aronsohn RS, Whitmore H, Van Cauter E, Tasali E. Impact of untreated obstructive sleep apnea on glucose control in type 2 diabetes. Am J Respir Crit Care Med. 2010;181:507-13.

9. He Q, Yang Q, Zhou Q, et al. Effects of varying degrees of intermittent hypoxia on proinflammatory cytokines and adipokines in rats and 3T3-L1 adipocytes. PLoS One. 2014;9(1):e86326.

10. Kim HW, Lee JE, Cha JJ, et al. Fibroblast growth factor 21 improves insulin resistance and ameliorates renal injury in $\mathrm{db} / \mathrm{db}$ mice. Endocrinology. 2013;154:3366-76.

11. Campos $\mathrm{I}$, Chan $\mathrm{L}$, Zhang $\mathrm{H}$, et al. Intradialytic hypoxemia in chronic hemodialysis patients. Blood Purif. 2016;41(1-3):177-87.

12. Jian WX, Peng WH, Jin J, et al. Association between serum fibroblast growth factor 21 and diabetic nephropathy. Metab Clin Exp. 2012;61:853-9.

13. Chinese Medical Association Diabetes Branch. China guidelines for type 2 diabetes prevention (2017 edition). Chin J Diabetes. 2018;10(1):4-67.

14. Working Group on Obesity in China. Guidelines for prevention and control of overweight and obesity in adults in China. J Nutr. 2004;26(1):1-4.

15. Microvascular Complications Group of Diabetes Branch of Chinese Medical Association. Expert consensus on prevention and treatment of diabetic nephropathy (2014 edition). Chin J Diabetes Mellitus. 2014;6(11):792-801.

16. Mather K, Kim C, Christophi C, et al. Steroid sex hormones, sex hormone binding globulin and diabetes incidence in the Diabetes Prevention Program. J Clin Endocrinol Metabol. 2015;2015:jc.2015-328.

17. Hui-Ming S, Dan X, Jia LI, et al. Clinical value of ultrasonic quantitative detection of liver fat content in patients with type 2 diabetes mellitus. Chin J Diabetes. 2014;22(11):966-9.

18. Tahrani AA. Obstructive sleep apnoea in diabetes: does it matter? Diab Vasc Dis Res. 2017;7:1479164117714397.

19. Mao S, Ren X, Zhang J. The emerging role of fibroblast growth factor 21 in diabetic nephropathy. J Recept Signal Transduct Res. 2016;36(6):1-7.
20. Wen-Juan C, Yang LI, Lan M, et al. The value of urinary albumin-to-creatinine ratio in the early diagnosis of diabetic kidney disease. Chin J Lab Diagnosis. 2013;17(9):1650-3.

21. Kaplan MJ. Cardiovascular complications of rheumatoid arthritis: assessment, prevention, and treatment. Rheum Dis Clin N Am. 2010;36(2):405-26.

22. Faulx MD, Storferisser A, Kirchner $H L$, et al. Obstructive sleep apnea is associated with increased urinary albumin excretion. Sleep. 2007;30(7):923.

23. Buyukaydin B, Akkoyunlu ME, Kazancioglu R, et al. The effect of sleep apnea syndrome on the development of diabetic nephropathy in patients with type 2 diabetes. Diabetes Res Clin Pract. 2012;98:1.

24. Taleb N, Salti H, Al-Mokaddam M, et al. Prevalence and determinants of albuminuria in a cohort of diabetic patients in Lebanon. Ann Saudi Med. 2008;28(6):420-5.

25. Zhang R, Guo X, Guo L, et al. Prevalence and associated factors of obstructive sleep apnea in hospitalized patients with type 2 diabetes in Beijing. China J Diabetes. 2015;7(1):16-23.

26. Furukawa S, Saito I, Yamamoto S, et al. Nocturnal intermittent hypoxia as an associated risk factor for microalbuminuria in Japanese patients with type 2 diabetes mellitus. Eur J Endocrinol. 2013;169(2):239-46.

27. Sim JJ, Rasgon SA, Derose SF. Review article: managing sleep apnoea in kidney diseases. Nephrology. 2010;15(2):146-52.

28. Hall IE, Kashgarian M, Moeckel GW, et al. Resolution of proteinuria in a patient with focal segmental glomerulosclerosis following BiPAP initiation for obesity hypoventilation syndrome [J]. Clin Nephrol. 2012;77(1):62.

29. Lin Z, Zhou Z, Liu Y, et al. Circulating FGF21 levels are progressively increased from the early to end stages of chronic kidney diseases and are associated with renal function in Chinese. PLoS One. 2011;6:e18398.

30. Liu Y, Wang C, Wang Y, et al. Cobalt chloride decreases fibroblast growth factor-21 expression dependent on oxidative stress but not hypoxia-inducible factor in Caco- 2 cells. Toxicol Appl Pharmacol. 2012;264(2):212-21. 